\title{
Recessive C10orf 2 mutations in a family with infantile-onset spinocerebellar ataxia, sensorimotor polyneuropathy, and myopathy
}

\author{
Mi-Hyun Park • Hae-Mi Woo • Young Bin Hong • Ji Hoon Park • \\ Bo Ram Yoon • Jin-Mo Park • Jeong Hyun Yoo • Heasoo Koo • \\ Jong-Hee Chae • Ki Wha Chung • Byung-Ok Choi • Soo Kyung Koo
}

Received: 19 December 2013 / Accepted: 25 April 2014 / Published online: 10 May 2014

(C) The Author(s) 2014. This article is published with open access at Springerlink.com

\begin{abstract}
Recessive mutations in chromosome 10 open reading frame 2 (C10orf2) are relevant in infantile-onset spinocerebellar ataxia (IOSCA). In this study, we investigated the causative mutation in a Korean family with combined phenotypes of IOSCA, sensorimotor polyneuropathy, and myopathy. We investigated recessive mutations in a Korean family with two individuals affected by IOSCA. Causative mutations were investigated using whole exome sequencing. Electrophysiological analyses and muscle and nerve biopsies were performed, along with magnetic resonance imaging (MRI) of the brain and lower extremities. Compound heterozygous mutations c. $1460 \mathrm{C}>\mathrm{T}$ and c.1485-1G $>\mathrm{A}$ in C10orf2 were identified as causative of IOSCA. Skeletal muscle showed mitochondrial DNA (mtDNA) deletions. Both patients showed a period of normal development until 1215 months, followed by ataxia, athetosis, hearing loss, and intellectual disability. Electrophysiological findings indicated
\end{abstract}

Electronic supplementary material The online version of this article (doi:10.1007/s10048-014-0405-1) contains supplementary material, which is available to authorized users.

M.-H. Park · H.-M. Woo • S. K. Koo $(\bowtie)$

Division of Intractable Diseases, Center for Biomedical Sciences,

National Institute of Health, Osong Health Technology

Administration Complex 643, Yeonje-ri, Osong-eup,

Cheongwon-gun, Chungcheongbuk-do 363-951, South Korea

e-mail: skkoo@nih.go.kr

Y. B. Hong • B.-O. Choi $(\square)$

Department of Neurology, Samsung Medical Center, Sungkyunkwan

University School of Medicine, 50 Ilwon-dong Gangnam-Gu,

Seoul 135-710, South Korea

e-mail: bochoi@skku.edu

J. H. Park $\cdot$ B. R. Yoon $\cdot$ K. W. Chung

Department of Biological Science, Kongju National University,

Gongju, South Korea motor and sensory polyneuropathies. Muscle biopsy revealed variations in the size and shape of myofibers with scattered, small, and angulated degenerating myofibers containing abnormal mitochondria; these observations are consistent with myopathy and may be the result of mtDNA deletions. Sural nerve biopsy revealed an axonal neuropathy. High-signalintensity lesions in the middle cerebellar peduncles were correlated with clinical severity, and MRI of the lower legs was compatible with the hypothesis of length-dependent axonal degeneration. We identified novel compound heterozygous mutations of the C10orf2 gene as the cause of IOSCA with sensorimotor polyneuropathy and myopathy. Signs of motor neuropathy and myopathy were discovered for the first time in IOSCA patients with C10orf2 mutations. These results suggest that the clinical spectrum of IOSCA caused by C10orf 2 mutations may be more variable than previously reported.

J.-M. Park

Department of Neurology, Ewha Womans University School of Medicine, Seoul, South Korea

J. H. Yoo

Department of Radiology, Ewha Womans University School of Medicine, Seoul, South Korea

H. Koo

Department of Pathology, Ewha Womans University School of Medicine, Seoul, South Korea

J.-H. Chae

Department of Pediatrics, Seoul National University College of Medicine, Seoul, South Korea 
Keywords C10orf2 - Whole exome sequencing (WES) . Infantile-onset spinocerebellar ataxia (IOSCA) - Neuropathy · Myopathy $\cdot$ Mitochondria

\begin{tabular}{|c|c|}
\hline \multicolumn{2}{|c|}{ Abbreviations } \\
\hline IOSCA & Infantile-onset spinocerebellar ataxia \\
\hline adPEO & $\begin{array}{l}\text { Autosomal dominant progressive external } \\
\text { ophthalmoplegia }\end{array}$ \\
\hline CMT & Charcot-Marie-Tooth disease \\
\hline MFs & Myelinated fibers \\
\hline WES & Whole exome sequencing \\
\hline mtDNA & Mitochondrial DNA \\
\hline FDS & Functional disability scale \\
\hline CMTNS & CMT neuropathy score \\
\hline HMSN & Hereditary motor and sensory neuropathy \\
\hline NCSs & Nerve conduction studies \\
\hline MNCVs & Motor nerve conduction velocities \\
\hline CMAPs & Compound muscle action potentials \\
\hline SNCVs & Sensory nerve conduction velocities \\
\hline $\begin{array}{l}\text { NADH- } \\
\text { TR }\end{array}$ & NADH-tetrazolium reductase \\
\hline SDH & Succinate dehydrogenase \\
\hline $\mathrm{COX}$ & Cytochrome oxidase \\
\hline ATPase & Adenosine triphosphatase \\
\hline
\end{tabular}

\section{Introduction}

Mutations in nuclear genes involved in mitochondrial DNA (mtDNA) maintenance are increasingly associated with a wide range of clinical phenotypes including encephalopathy, progressive external ophthalmoplegia (PEO), ataxia, myopathy, and Parkinson's disease [1, 2]. Mutations in DNA polymerase gamma (POLG), DNA helicase Twinkle (C10orf2), or mitochondrial transcription factor A (TFAM) have been linked to the deterioration of mtDNA [3].

Chromosome 10 open reading frame 2 (C10orf2), also known as Twinkle or PEO1, encodes the mitochondrial helicase Twinkle. Defects in C10orf2 lead to the accumulation of multiple deletions in the mtDNA of affected tissues and an associated respiratory chain defect [4]. Mutations in the C10orf2 gene have been shown to play a role in multiple autosomal recessive diseases including infantile-onset spinocerebellar ataxia (IOSCA), hepatocerebral syndrome, and autosomal dominant PEO (adPEO) [5]. However, it remains unclear why some autosomal recessive mutations result in IOSCA while others cause hepatocerebral syndrome.

The clinical symptoms of IOSCA (OMIM\# 271245) are characterized by a period of normal development, followed by onset of ataxia, hypotonia, loss of deep tendon reflexes, and athetosis between 9 and 18 months [6]. Later stages of disease are characterized by ophthalmoparesis, sensorineural hearing loss, epilepsy, and primary hypogonadism in female patients. Electromyographic findings are characterized by axonal sensory neuropathy; however, no reports exist describing an IOSCA patient harboring motor neuropathy [5-7]. Although adPEO patients with C10orf2 mutations usually demonstrate mtDNA deletions in muscle [8,9], IOSCA patients show no signs of mtDNA deletion. Muscle biopsies of IOSCA patients revealed only nonspecific fiber-type groupings $[5-7,10]$. In contrast to the more well-described adPEO, a limited number of reports have been written on IOSCA.

We report the clinical, histopathological, and genetic defects observed with IOSCA, sensorimotor polyneuropathy, and myopathy, which were associated with novel compound heterozygous mutations in C10orf2.

\section{Materials and methods}

\section{Subjects}

This study examined four members of a Korean family (family ID: FC417), two of whom were affected by IOSCA (Fig. 1a). Phenotypic analysis of this family suggested an autosomal recessive mode of inheritance as both children exhibited symptoms of the disease, while both parents were unaffected. No affected individuals were identified among close relatives of the family. Written informed consent was obtained from all participants and from a parent of the participants younger than 18 years of age, according to the protocol approved by the Institutional Review Board of Ewha Womans University, Mokdong Hospital, and the Korea National Institutes of Health (KNIH).

\section{Clinical and electrophysiological assessments}

Two independent neurologists evaluated the patients by recording a detailed history of motor and sensory impairments, deep tendon reflexes, and muscle atrophy. Flexor and extensor muscle strength were assessed manually using the Medical Research Council (MRC) scale. To determine physical disability, we used two scales, a functional disability scale (FDS) [11] and a Charcot-Marie-Tooth neuropathy score (CMTNS) [12]. Disease severity was determined for each patient using a nine-point FDS. Sensory impairments were assessed in terms of the magnitude and severity of pain, temperature, vibration, and position perceptions.

Nerve conduction studies (NCSs) were performed using surface electrodes in the median, ulnar, peroneal, tibial, and sural nerves. Motor nerve conduction velocities (MNCVs) of the median and ulnar nerves were determined by providing stimulation at the elbow and wrist while recording compound muscle action potentials (CMAPs) over the abductor pollicis brevis and adductor digiti quinti, respectively. In the same 
a

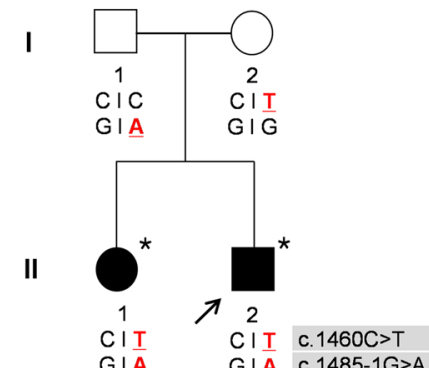

b

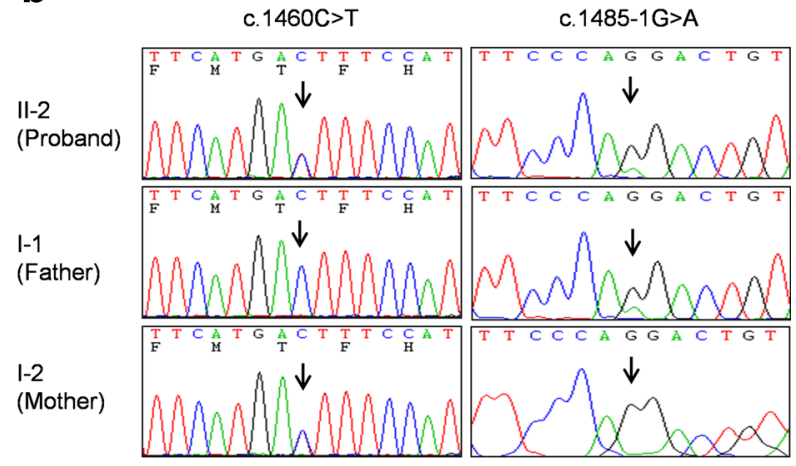

C

Homo sapiens
Mus musculus
Rattus norvegicus
Bos taurus
Macaca mulatta
Gallus gallus
Pan paniscus
Equus caballus
Felis catus
Canis lupus familiar

c. $1460 \mathrm{C}>\mathrm{T}$

HWADREEDLPLYEMTEHGQQS IRTVIDTM EWADREEDLPLYEMTEHGQQSIRSVIDTM EWADREEDLPLYEMTEHGQQSIRSVIDTM EWADREEDLPLYEMTEHGQQSIRTVIDTM HWADREEDLPLYEMTEHGQOSIRTVIDTM EWADREEDLPLYEMTEHGQQNIKTVLDTM HWADREEDLPLYFMTEHGQQSIRTVIDTM EWADREEDLPLYEMTEHGQQSIRTVIDTM EWADREEDLPLYEMTEHGQQSIRTVIDTM EWADREEDLPLYEMTEHGQQSIRTVIDTM d

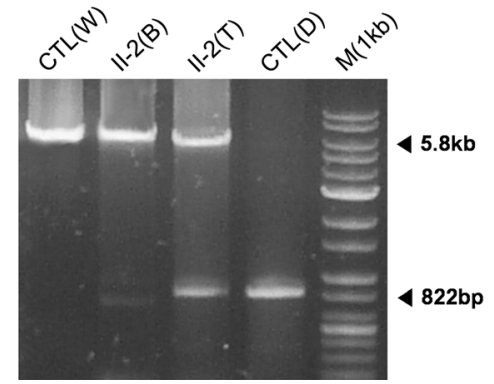

Fig. 1 Pedigree, sequencing of C10orf2 gene mutations, and mtDNA deletions in a Korean family. a Pedigree of family FC417. Open symbols represent unaffected individuals and filled symbols affected individuals. Proband is indicated by an arrow. Asterisks indicate individuals whose DNA was used for exome sequencing. C10orf2 sequences are indicated below each family member. b Sequencing chromatograms of C10orf2 mutations. Vertical arrows indicate the mutation site. c Conservation of the amino acid sequence in the p.T487I mutation region. The mutation site is highly conserved among vertebrate species: $H$. sapiens, NP_068602.2; M. musculus, NP_722491.2; R. norvegicus,
NP_001101069.1; B. taurus, NP_001091933.1; M. mulatta, NP_001181370.1; G. gallus, NP_001026515.1; P. paniscus, XP_003825527.1; E. caballus, XP_001499990.1; F. catus, XP_003994378.1; and C. lupus familiaris, XP_003433659.1. d Identification of a 4,977-bp common deletion in mtDNA. The 4,977-bp large deletion (m.8470_13446del4977) was detected using the long template PCR method. $C T L(W)$, wild type; $C T L(D)$, common deletion in adPEO; $I I-2(B)$, DNA from the blood of patient II-2; II-2(T), DNA from the vastus lateralis muscle; and $M(1 \mathrm{~kb}), 1-\mathrm{kb}$ DNA ladder manner, the MNCVs of the peroneal and tibial nerves were determined by stimulation at the knee and ankle while recording CMAPs over the extensor digitorum brevis and adductor hallucis, respectively. Sensory nerve conduction velocities (SNCVs) were obtained over a finger-wrist segment from the median and ulnar nerves by orthodromic scoring; SNCVs were also recorded for sural nerves. Needle electromyography (EMG) was performed on bilateral proximal and distal limb muscles of the upper and lower extremities. Visual evoked potentials and brainstem auditory evoked potentials in two patients (II-1 and II-2) were done.

Vastus lateralis muscle biopsy

Histopathological analysis of the skeletal muscle was performed on a 21-year-old patient (patient II-2). The muscle biopsy was taken from the left vastus lateralis muscle under local anesthesia. Frozen sections were stained with hematoxylin and eosin and Gomori's trichrome stain and for nicotinamide adenine dinucleotide (NADH)-tetrazolium reductase (NADH-TR), succinate dehydrogenase (SDH), and cytochrome oxidase (COX). Adenosine triphosphatase (ATPase) activity was assessed under different $\mathrm{pH}$ conditions (ATPase at $\mathrm{pH} 9.4$ followed by preincubation at $\mathrm{pH} 4.6$ and 4.3). An ultrastructural analysis was also performed.

\section{Distal sural nerve biopsy}

Histopathological analysis of the distal sural nerve biopsy was performed at 19 years of age (patient II-2). In addition to light microscopic examination, electron microscopic observations were made using specimens fixed in $2 \%$ glutaraldehyde in $0.025 \mathrm{M}$ cacodylate buffer ( $\mathrm{pH} 7.4$ ) and processed for semithin and ultra-thin studies. Semi-thin sections were stained with toluidine blue for evaluation by light microscopy. Ultrathin sections $(60-65 \mathrm{~nm})$ were contrasted with uranyl acetate and lead citrate for ultrastructural studies (H-7650, Hitachi, Japan). The density of myelinated fibers (MFs), axonal diameter, myelin thickness, and the g-ratio of MFs were determined from semi-thin transverse sections using a computer-assisted image analyzer (AnalySIS, Soft Imaging System, Germany). 
Magnetic resonance imaging (MRI) of the brain and lower extremities

The brain, thigh, and lower leg of patients II-1 and II-2 were evaluated by MRI using a 1.5-T system (Siemens Vision, Siemens, Germany). Whole brains were scanned using a slice thickness of $5 \mathrm{~mm}$ and a $2-\mathrm{mm}$ interslice gap, to produce 16 axial images. The imaging protocol consisted of T2-weighted spin echo (SE) (repetition time (TR)/echo time $(\mathrm{TE})=4,700 /$ $120 \mathrm{~ms}$ ), T1-weighted SE (TR/TE=550/12 ms), and fluidattenuated inversion recovery (FLAIR) $(\mathrm{TR} / \mathrm{TE}=9,000 /$ $119 \mathrm{~ms}$; inversion time $=2,609 \mathrm{~ms}$ ) images. Thigh and lower leg (calf) muscle imaging was performed in axial [field of view $(\mathrm{FOV})=24-32 \mathrm{~cm}$; slice thickness $=6 \mathrm{~mm}$; slice gap $=0.5-1.0 \mathrm{~mm}]$ and coronal planes $(\mathrm{FOV}=38-40 \mathrm{~cm}$; slice thickness $=4-5 \mathrm{~mm}$; slice gap $=0.5-1.0 \mathrm{~mm})$. The following protocol was used: T1-weighted SE (TR/TE $=570-650 / 14$ 20, 512 matrixes), T2-weighted $\mathrm{SE}(\mathrm{TR} / \mathrm{TE}=2,800-4,000$ / 96-99; 512 matrixes), and fat-suppressed T2-weighted SE (TR/TE $=3,090-4,900 / 85-99,512$ matrixes).

\section{Whole exome sequencing}

Whole exome sequencing was performed for two individuals (II-1 and II-2). Exome capture/enrichment of $44 \mathrm{Mb}$ was performed using the Human SeqCap EZ Human Exome Library v2.0 (Roche/NimbleGen, Madison, WI). Captured DNA was amplified and sequenced on the Solexa GAIIx (for II-1) and HiSeq2000 (II-2) Genome Analyzers (Illumina, San Diego, CA). Paired-end sequences were mapped to the reference human genome (GRCh37, UCSC hg19) using BWA (http://bio-bwa.sourceforge.net/). The variant calling process was run using SAMtools (http://samtools.sourceforge.net/). For functional annotation and genetic filtering, variants were submitted to ANNOVAR (http:/www.openbioinformatics. org/annovar/). Variants were filtered using exome data from 35 Korean patients, including data from 29 CMT patients [13, 14] and 6 deaf patients [15], along with Korean-specific variants deposited in the TIARA database [16]. To confirm candidate variants in additional Korean controls, we used exome data from an additional 648 patient samples.

Capillary sequencing was performed to confirm candidate variants. PCR products were sequenced using an ABI 3730 automatic genetic analyzer (Applied Biosystems, Foster City, CA). Mutations were described according to the Human Genome Variant Society (HGVS) nomenclature (http://www. hgvs.org/mutnomen) with nucleotide numbering based on the mRNA sequence (NM 021830.4) of C10orf2 (http:// www.ncbi.nlm.nih.gov/nuccore/NM_021830). Segregation analysis of mutations with disease phenotypes was performed for all family members.
Mitochondrial DNA analysis

A deletion of 4,977 bp (m.8470_13446del4977) in mtDNA, frequently referred to as the "common deletion," was tested in DNA extracted from whole blood and the vastus lateralis muscle of patient II-2, using an Expand Long Template PCR System (Roche, Germany). PCR primers covered nucleotides 8,225-8,247 (forward) and 13,707-13,729 (reverse) of the revised Cambridge reference sequence (NC_012920.1).

\section{Results}

Identification of the novel mutation in the C10orf2 gene

We performed whole exome sequencing on an autosomal recessive IOSCA family with sensorimotor polyneuropathy and myopathy to identify mutations causally associated with disease onset (Fig. 1a). Exome sequencing results of two individuals are shown in Table 1. A total of 58,262 and 61,340 variants were identified in each of the two individuals, respectively. Of these, a total of 10,714 and 9,543 variants were identified as functionally significant, resulting in missense, nonsense, splice, or indel mutations. After filtering out all dbSNP132 variants, we were able to reduce the numbers of

Table 1 Exome sequencing results for two affected individuals of family FC417

\begin{tabular}{lll}
\hline Patients & II-1 & II-2 \\
\hline Mappable yield (bp) & $5,206,019,945$ & $5,937,912,039$ \\
On-target yield (bp) & $3,248,319,559$ & $3,393,707,908$ \\
\% Coverage of targeted region & $97.9 \%$ & $97.0 \%$ \\
$\quad(44 \mathrm{Mb})>1 \times$ & & \\
$\%$ Coverage of targeted region & $94.0 \%$ & $90.9 \%$ \\
$\quad(44 \mathrm{Mb})>10 \times$ & & \\
Mean read depth of targeted region & $73.8 \times$ & $77.1 \times$ \\
Mean read depth of called variant $\geq 5 \times$ & $58.9 \times$ & $55.4 \times$ \\
Number of SNPs & 50,733 & 53,119 \\
Number of indels & 7,529 & 8,221 \\
Total variants & 58,262 & 61,340 \\
Total coding, splicing variants & 21,084 & 19,516 \\
Missense, nonsense, splice, & 10,714 & 9,543 \\
$\quad$ indel variants & & \\
After dbSNP132 filtering & 1,231 & 824 \\
Shared variants between the & 365 & \\
$\quad$ two individuals & & \\
After filtering with Korean control & 146 & \\
$\quad$ exomes &
\end{tabular}

${ }^{\mathrm{a}}$ Exome data of 35 Koreans from other reports and the Korean genomes database, TIARA 
functional variants to 1,231 and 824 , respectively, with 365 variants shared between the two individuals. Additional filtering was performed by excluding all variants identified in a cohort of 35 Korean control exomes.

Based upon these analyses, we were able to identify compound heterozygous variants in two genes, C10orf2 and leukocyte immunoglobulin-like receptor, superfamily B (LILRB1) (Table 1). We compared the four variants in C10orf2 (Chr10:102749617 and Chr10:102750192) and LILRB1 (Chr19:55143632 and Chr19:55145125) with those identified in the 248 exome dataset. Only two variants in gene C10orf2 were absent from the 248 exome dataset (Supplementary Table 1); these variants were also absent in a larger collection of in-house exome data $(n=400)$. We confirmed that both C10orf2 heterozygous mutations c.1460C $>$ T (p.T487I) and c.1485-1G $>$ A showed complete segregation with the disease phenotype within the family (Fig. 1a, b). The patients' father carried a c.1485-1G $>$ A splicing-site mutation, while the mother carried the c. $1460 \mathrm{C}>\mathrm{T}$ missense mutation. The p.T487I mutation was located at a highly conserved location (Fig. 1c) and was predicted to be damaging, according to in silico analyses using SIFT, PolyPhen-2, and MUpro (Table 2). As several mutations in C10orf2 have been implicated in recessive IOSCA [6-8], we therefore concluded that the two novel compound heterozygous mutations in C10orf2 described here were the underlying cause of IOSCA in this family.

mtDNA deletion in muscle

As mutations in C10orf2 are associated with deletions in mtDNA, we examined the mtDNA of patient 1 (II-2); this revealed a large heteroplasmic common deletion of 4,977 bp (m.8470_13446del4977) in skeletal muscle, whereas few or no deletions were identified in blood mtDNA (Fig. 1d).

\section{Clinical manifestations}

The clinical features of patients with C10orf2 mutations are described in Table 3. Patient II-2 (Fig. 2a, proband) was the second child of healthy, non-consanguineous Korean parents. He was born at full term, and the prenatal and neonatal history was unremarkable. Early motor milestones were not significantly delayed until 1 year of age, when his parents noticed that he had become clumsy and spoke less often than before. He was in a wheelchair by the age of 14 years. At age 17, we performed MRI of the brain as the result of an ataxia. By 20 years of age, ataxia had become more severe, and a followup MRI was performed. Neurological examination at 21 years of age revealed severe distal muscle atrophy, pes cavus, and scoliosis. Symptoms of ophthalmoplegia were not observed. An ophthalmologist examined the patient, including dilated fundus examination, and he confirmed that no optic atrophy was present. Vibration and position senses were more severely disturbed than pain and temperature senses. Deep tendon reflexes were absent in all extremities. FDS and CMTNS scores were 7 and 26, respectively, with both scores classifying this patient as severely disabled. He also exhibited substantial intellectual disability, with an intelligence quotient score of 54. A positron emission tomography (PET) scan of patient II-2 showed decreased metabolic changes throughout the brain. An echocardiogram was normal, and there was no evidence of migraine, seizure, or psychiatric symptoms. An electroencephalogram revealed a normal sleep record without epileptiform discharges. Secondary sex characteristics were well developed, with no signs of hypogonadism. His blood lactate level was $17.0 \mu \mathrm{mol} / \mathrm{L}$ (reference interval 4.5-14.4 $\mu \mathrm{mol} / \mathrm{L}$ ), pyruvate level was $0.7 \mu \mathrm{mol} / \mathrm{L}$ (reference interval $0.3-0.9 \mu \mathrm{mol} / \mathrm{L})$, and creatine kinase level was $155 \mu \mathrm{mol} / \mathrm{L}$ (reference value $<185 \mu \mathrm{mol} / \mathrm{L}$ ), all well within normal ranges. Liver transaminase levels were normal: the AST level was $22 \mu / \mathrm{L}$ (reference value $<40 \mu / \mathrm{L}$ ), and the alanine transaminase (ALT) was $27 \mu / \mathrm{L}$ (reference value $<40 \mu / \mathrm{L}$ ).

Patient II-1 (Fig. 2) was the elder sister of patient II-2 and was born at full term after an uneventful pregnancy. Early motor milestones were not significantly delayed until 15 months, when her parents noticed that she had become clumsy. She was first examined at 3 years of age due to ataxia and athetoid movements; however, symptoms of ophthalmoplegia were not observed. An ophthalmologist examined the patient, and he confirmed that no optic atrophy was present. A hearing deficit was found, and by school age, she was completely deaf and began communicating with sign language. She became

Table 2 C10orf2 heterozygous mutations associated with IOSCA with sensorimotor polyneuropathy and myopathy

\begin{tabular}{|c|c|c|c|c|c|c|}
\hline \multirow[t]{2}{*}{ Gene } & \multicolumn{2}{|l|}{ Mutation } & \multirow[t]{2}{*}{ In controls } & \multicolumn{3}{|c|}{ In silico analysis } \\
\hline & Nucleotide & Amino acid & & SIFT & PolyPhen-2 & MUpro \\
\hline \multirow[t]{2}{*}{ C10orf2 } & c. $1460 \mathrm{C}>\mathrm{T}$ & p.T487I & $0 / 648$ & $0.02^{\mathrm{a}}$ & $1.00^{\mathrm{a}}$ & $-0.566^{\mathrm{a}}$ \\
\hline & c. $1485-1 \mathrm{G}>\mathrm{A}$ & Splicing acceptor & $0 / 648$ & - & - & - \\
\hline
\end{tabular}

${ }^{a}$ Values indicate prediction of significant effect on protein structure or function 
Table 3 Comparison of the clinical phenotypes of IOSCA patients with mutations in the C10orf2 gene

\begin{tabular}{|c|c|c|c|c|}
\hline Patients (ethnicity) & Korean & English & Finnish & Turkish \\
\hline Mutations & {$[\mathrm{T} 487 \mathrm{I}]+[1485-1 \mathrm{G}>\mathrm{A}]$} & {$[\mathrm{P} 83 \mathrm{~S}]+[\mathrm{R} 463 \mathrm{~W}]$} & {$[\mathrm{Y} 508 \mathrm{C}]+[\mathrm{Y} 508 \mathrm{C}],[\mathrm{Y} 508 \mathrm{C}]+[\mathrm{A} 318 \mathrm{~T}]$} & {$[\mathrm{L} 456 \mathrm{~V}]+[\mathrm{L} 456 \mathrm{~V}]$} \\
\hline Number of patients & 2 & 1 & 23 & 2 \\
\hline Onset age (years) & $>1$ & $>1$ & $>1$ & $>1$ \\
\hline Normal early milestones & Yes & Yes & Yes & Yes \\
\hline Athetosis & Yes & Yes & Yes & Yes \\
\hline Areflexia & Yes & Yes & Yes & Yes \\
\hline Pes cavus & Yes & NA & Yes & Yes \\
\hline Scoliosis & Yes & NA & Yes & NA \\
\hline Hearing loss & Yes & NA & Yes & Yes \\
\hline Psychomotor retardation & Yes & NA & Yes & Yes \\
\hline Ophthalmoplegia & No & Yes & Yes & Yes \\
\hline Liver involvement & No & No & Yes & No \\
\hline Kidney involvement & No & No & No & No \\
\hline Nerve conduction study & Sensorimotor polyneuropathy & Sensory neuropathy & Sensory neuropathy & Sensory neuropathy \\
\hline Electromyography & Abnormal & Abnormal & Abnormal & Abnormal \\
\hline Electroencephalography & Normal & Abnormal & Abnormal $^{\mathrm{a}}$ & Normal \\
\hline mtDNA depletion & NA & NA & Brain, liver & NA \\
\hline mtDNA deletion & Yes & NA & No & NA \\
\hline Liver enzyme & Normal & Abnormal $^{\mathrm{b}}$ & Abnormal $^{\mathrm{c}}$ & Normal \\
\hline Creatine kinase & Normal & NA & Normal & NA \\
\hline Lactate & Elevated & Elevated $^{\mathrm{d}}$ & Normal & Normal \\
\hline Pyruvate & Normal & NA & Normal & NA \\
\hline Lactate/pyruvate ratio & $45.6,24.3$ & NA & NA & NA \\
\hline Respiratory chain & Normal & Normal & Normal & NA \\
\hline Brain MRI & Abnormal & Abnormal & Abnormal & Abnormal \\
\hline Lower extremity MRI & Abnormal & NA & NA & NA \\
\hline Muscle biopsy & Myopathy, type grouping & Type grouping & Type grouping & Type grouping \\
\hline Nerve biopsy & Axonal loss & Axonal loss & Axonal loss & NA \\
\hline References & This study & [7] & {$[5,10,22,23]$} & {$[6]$} \\
\hline
\end{tabular}

$N A$ not available

${ }^{\text {a }}$ Abnormal after epileptic encephalopathy

${ }^{\mathrm{b}}$ When her symptoms worsen, liver enzymes were elevated

${ }^{\mathrm{c}}$ Abnormal in patients with compound heterozygote mutation

${ }^{\mathrm{d}}$ Inconsistently elevated in capillary lactate, while normal in CSF lactate

wheelchair bound by the age of 14 years. When we examined her at 23 years of age, she displayed muscle weakness and atrophy of the bilateral distal muscles of the upper and lower limbs. Loss of sensory and tendon reflexes was similar to those of her younger brother. FDS and CMTNS scores were 7 and 28, respectively, both in the severe disability category. Pes cavus and scoliosis were observed. Like her brother, she also exhibited significant intellectual disability. An echocardiogram was normal, and there was no evidence of migraine, seizure, or psychiatric symptoms. An electroencephalogram revealed a normal sleep record without epileptiform discharges. Secondary sex characteristics were poorly developed, and she never menstruated. Her lactate level was $41.0 \mu \mathrm{mol} / \mathrm{L}$, pyruvate level was $0.9 \mu \mathrm{mol} / \mathrm{L}$, and creatine kinase level was $71 \mu \mathrm{mol} / \mathrm{L}$, all within normal ranges. Liver transaminase levels were normal: the AST level was $23 \mu / \mathrm{L}$, and the ALT level was $38 \mu / \mathrm{L}$. The father (I-1, 50 years of age) and mother (I-2, 47 years of age) were found to be normal by careful clinical and electrophysiological examinations.

\section{Electrophysiological findings}

The electrophysiological features of patients II- 1 and II-2 are described in Table 4. NCSs were performed three times, at 
Fig. 2 Images of patients II-2 (a 21 years of age) and II-1 (b 23 years of age). a Patient II-2 was unable to walk and has been wheelchair-bound since 5 years of age. Moderate-to-severe atrophies of the lower limb muscles and high-arched feet were evident. b Patient II-1 was wheelchairbound by 7 years of age. Atrophies of the lower leg and hand muscles were prominent. Substantial ankle joint contractures and foot deformities were also evident
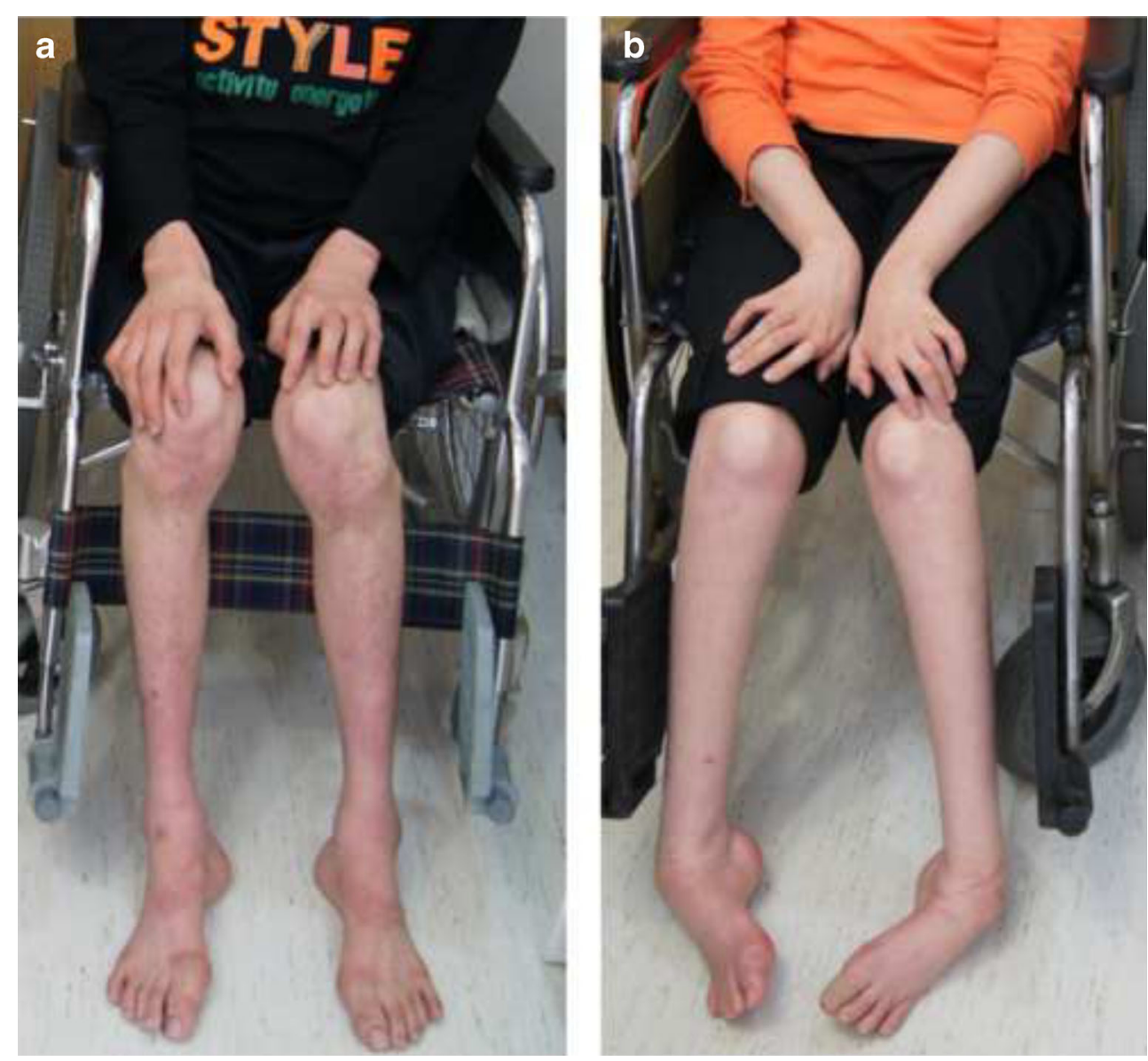

19, 20, and 21 years of age for patient II-2, and at 22 years of age for patent II-1. MNCVs of the median, ulnar, peroneal, and tibial nerves were all decreased. In patient II-2, peroneal CMAPs were not elicited, and tibial CMAPs were reduced. Sensory nerve action potentials (SNAPs) of the median, ulnar, and sural nerves were absent in both patients. The interval changes over 3 years in patient II-2 revealed slow disease progression. Needle EMG showed fibrillation and positive sharp waves in the proximal and distal muscles. Delayed visual evoked potentials and brainstem auditory evoked potentials were decreased in both patients.

\section{Muscle biopsy findings}

Biopsy examination revealed variations in the size and shape of fibers, characterized by small, scattered, angulated degenerating myofibers (arrows), including abnormal condensed NADH-tetrazolium-positive cells (Fig. 3a, b). Electron microscopic examination confirmed the presence of degenerating myofibers with diffusely scattered autophagic vacuoles, distorted myofibrillar arrangement, abnormal membranous structures, and aggregated enlarged mitochondria with swelling, concentric cristae, and dense inclusions (Fig. 3c, d). ATPase reactions revealed groupings of both type 1 and type 2 myofibers across multiple $\mathrm{pH}$ concentrations (Fig. 3e, f). No differences were seen following cytochrome oxidase (Fig. 3g) or succinate dehydrogenase (Fig. 3h) staining.

\section{Nerve biopsy findings}

Light microscopic examination of longitudinal and cross sections of nerve fibers showed markedly decreased size of nerve fascicles with marked subperineurial edema and multifocal variable-sized Renaut bodies, which consists of loose EMA-positive spindle cells. Semi-thin transverse sections showed small MFs with almost complete loss of large MFs (Fig. 4a). Remaining MFs counted $5,101 / \mathrm{mm}^{2}$ (normal distal sural nerve in 21year-old male, $10,000 / \mathrm{mm}^{2}$ ). The range and average of diameter of MFs were $1.50-10.13$ and $3.87 \mu \mathrm{m}$, respectively (The range and average of diameter of MFs in a normal distal sural nerve of a 21-year-old male are 2.214.2 and $5.4 \mu \mathrm{m}$, respectively). The histogram of diameter sizes showed a unimodal distribution pattern; $19.5 \%$ of the MFs had a diameter less than $3 \mu \mathrm{m}$, 
Table 4 Electrophysiological features of IOSCA patients with compound heterozygous C10orf2 mutations

\begin{tabular}{|c|c|c|c|c|c|c|c|c|c|}
\hline \multirow[b]{2}{*}{ Age at exam (years) } & \multicolumn{2}{|c|}{ Patient II-1 } & \multicolumn{6}{|c|}{ Patient II-2 } & \multirow[t]{2}{*}{ Normal value } \\
\hline & 22 & & 19 & & 20 & & 21 & & \\
\hline Side & $\mathrm{Rt}$ & $\mathrm{Lt}$ & Rt & $\mathrm{Lt}$ & $\mathrm{Rt}$ & $\mathrm{Lt}$ & Rt & $\mathrm{Lt}$ & \\
\hline \multicolumn{10}{|l|}{ Median nerve } \\
\hline $\mathrm{TL}(\mathrm{ms})$ & 3.5 & 3.3 & 4.3 & 4.2 & 4.6 & 4.2 & 4.1 & 4.3 & $<3.9$ \\
\hline CMAP (mV) & 5.9 & 8.8 & 9.7 & 7.2 & 9.6 & 6.1 & 11.3 & 6.2 & $>6.0$ \\
\hline $\operatorname{MNCV}(\mathrm{m} / \mathrm{s})$ & 48.9 & 48.9 & 48.1 & 47.9 & 47.3 & 42.9 & 47.2 & 43.6 & $>50.5$ \\
\hline F-wave (ms) & 29.6 & 29.0 & 34.0 & 32.8 & 34.6 & 35.2 & 36.6 & 33.6 & $<28.0$ \\
\hline \multicolumn{10}{|l|}{ Ulnar nerve } \\
\hline TL (ms) & 2.7 & 2.6 & 2.9 & 3.4 & 3.3 & 3.5 & 2.6 & 3.1 & $<3.0$ \\
\hline CMAP (mV) & 11.1 & 8.6 & 10.1 & 9.0 & 10.8 & 7.7 & 9.9 & 7.3 & $>8.0$ \\
\hline $\operatorname{MNCV}(\mathrm{m} / \mathrm{s})$ & 48.9 & 47.8 & 49.0 & 47.2 & 45.5 & 43.1 & 45.5 & 45.5 & $>51.1$ \\
\hline F-wave (ms) & 30.0 & 29.2 & 32.8 & 33.0 & 34.6 & 35.0 & 37.4 & 34.2 & $<29.0$ \\
\hline \multicolumn{10}{|l|}{ Peroneal nerve } \\
\hline $\mathrm{TL}(\mathrm{ms})$ & 5.4 & 5.5 & $\mathbf{A}$ & $\mathbf{A}$ & $\mathbf{A}$ & $\mathbf{A}$ & $\mathbf{A}$ & $\mathbf{A}$ & $<5.3$ \\
\hline CMAP (mV) & 1.5 & 0.8 & $\mathbf{A}$ & $\mathbf{A}$ & $\mathbf{A}$ & $\mathbf{A}$ & $\mathbf{A}$ & $\mathbf{A}$ & $>1.6$ \\
\hline $\operatorname{MNCV}(\mathrm{m} / \mathrm{s})$ & 37.4 & 36.6 & $\mathbf{A}$ & $\mathbf{A}$ & $\mathbf{A}$ & $\mathbf{A}$ & $\mathbf{A}$ & $\mathbf{A}$ & $>41.2$ \\
\hline F-wave (ms) & 52.2 & 73.8 & $\mathbf{A}$ & $\mathbf{A}$ & $\mathbf{A}$ & $\mathbf{A}$ & $\mathbf{A}$ & $\mathbf{A}$ & $<49.0$ \\
\hline \multicolumn{10}{|l|}{ Tibial nerve } \\
\hline $\mathrm{TL}(\mathrm{ms})$ & 2.8 & 3.3 & 7.2 & 5.0 & 4.2 & 4.4 & 4.2 & 4.5 & $<5.4$ \\
\hline CMAP (mV) & 2.7 & 3.9 & 1.5 & 1.1 & 1.3 & 1.0 & 0.8 & 1.0 & $>6.0$ \\
\hline $\operatorname{MNCV}(\mathrm{m} / \mathrm{s})$ & 36.6 & 37.9 & 24.2 & 27.3 & 28.7 & 29.4 & 28.1 & 32.0 & $>41.1$ \\
\hline F-wave (ms) & 56.6 & 49.0 & 75.8 & $\mathbf{A}$ & 75.6 & 70.4 & 72.2 & 71.0 & $<52.1$ \\
\hline \multicolumn{10}{|l|}{ Median sensory nerve } \\
\hline SNAP $(\mu \mathrm{V})$ & $\mathbf{A}$ & $\mathbf{A}$ & $\mathbf{A}$ & $\mathbf{A}$ & $\mathbf{A}$ & $\mathbf{A}$ & $\mathbf{A}$ & $\mathbf{A}$ & $>8.8$ \\
\hline $\mathrm{SNCV}(\mathrm{m} / \mathrm{s})$ & $\mathbf{A}$ & $\mathbf{A}$ & $\mathbf{A}$ & $\mathbf{A}$ & $\mathbf{A}$ & $\mathbf{A}$ & $\mathbf{A}$ & $\mathbf{A}$ & $>39.3$ \\
\hline \multicolumn{10}{|l|}{ Ulnar sensory nerve } \\
\hline SNAP $(\mu \mathrm{V})$ & $\mathbf{A}$ & $\mathbf{A}$ & $\mathbf{A}$ & $\mathbf{A}$ & $\mathbf{A}$ & $\mathbf{A}$ & $\mathbf{A}$ & $\mathbf{A}$ & $>7.9$ \\
\hline $\mathrm{SNCV}(\mathrm{m} / \mathrm{s})$ & $\mathbf{A}$ & $\mathbf{A}$ & $\mathbf{A}$ & $\mathbf{A}$ & $\mathbf{A}$ & $\mathbf{A}$ & $\mathbf{A}$ & $\mathbf{A}$ & $>37.5$ \\
\hline \multicolumn{10}{|l|}{ Sural nerve } \\
\hline SNAP $(\mu V)$ & $\mathbf{A}$ & $\mathbf{A}$ & $\mathbf{A}$ & $\mathbf{A}$ & $\mathbf{A}$ & $\mathbf{A}$ & $\mathbf{A}$ & $\mathbf{A}$ & $>6.0$ \\
\hline $\mathrm{SNCV}(\mathrm{m} / \mathrm{s})$ & $\mathbf{A}$ & $\mathbf{A}$ & $\mathbf{A}$ & $\mathbf{A}$ & $\mathbf{A}$ & $\mathbf{A}$ & $\mathbf{A}$ & $\mathbf{A}$ & $>32.1$ \\
\hline H-reflex (ms) & $\mathbf{A}$ & $\mathbf{A}$ & $\mathbf{A}$ & $\mathbf{A}$ & $\mathbf{A}$ & $\mathbf{A}$ & $\mathbf{A}$ & $\mathbf{A}$ & $<30.2$ \\
\hline
\end{tabular}

Bold characters indicate abnormal values. Normal conduction velocities: median motor nerve $\geq 50.5 \mathrm{~m} / \mathrm{s}$; ulnar nerve $\geq 51.1 \mathrm{~m} / \mathrm{s}$; and sural nerve $\geq 32.1 \mathrm{~m} /$ s. Normal amplitudes: median motor nerve $\geq 6.0 \mathrm{mV}$; ulnar nerve $\geq 8.0 \mathrm{mV}$; and sural nerve $\geq 6.0 \mu \mathrm{V}$

$A$ absent potentials, $T L$ terminal latency, $C M A P$ compound muscle action potential, $M N C V$ motor nerve conduction velocity, $S N A P$ sensory nerve action potential, $S N C V$ sensory nerve conduction velocity, $N P$ no potential

and $97.0 \%$ had a diameter less than $6 \mu \mathrm{m}$ (Fig. 4b). The $\mathrm{MF} \%$ area in this case was $6.46 \%$ (normal sural nerve of a 21-year-old male, $36.5 \%$ ). On electron microscopic examination, the remaining small MFs showed occasional excessive folding of myelin with very rare evidence of regeneration (clusters of regenerating fibers, small MFs with collars of Schwann cell processes containing small unmyelinated axons, mimicking a pseudo-onion bulb, or basal lamina onion bulb formation) (Fig. 4c, d). Endoneurial fibroblast proliferation and collagen deposition were evident.
Brain and lower extremity MRI findings

An MRI revealed hyperintense signal abnormalities in the brains of both patients (Fig. 5). In patient II-1, high-signalintensity lesions of the bilateral middle cerebellar peduncles were observed on T2-weighted images (Fig. 5a). In patient II-2, similar lesions were not found at the age of 17 years (Fig. 5b); however, a follow-up study performed 3 years later revealed bilateral symmetric highsignal-intensity lesions in the middle cerebellar peduncle (Fig. 5c), with a very similar appearance to those 
Fig. 3 Histopathological characterization of the vastus lateralis muscle (patient II-2). a-b NADH-TR staining revealed a scattering of small, angulated, or elongated myofibers with condensed positive reactions (arrows). c-d Electron micrographs revealed degenerating myofibers containing diffusely scattered autophagic vacuoles, distorted myofibrillar arrangement, abnormal membranous structures, and aggregated enlarged mitochondria with swelling, concentric cristae, and dense inclusions. e-f ATPase staining at $\mathrm{pH} 9.4$ revealed grouping of type 1 and type 2 myofibers. $\mathbf{g}$ Cytochrome oxidase (COX) staining did not show COXnegative fibers. $\mathbf{h}$ Increased succinate dehydrogenase staining was not observed. Original magnifications: $\mathbf{a} \times 100 ; \mathbf{b} \times 200$; $\times 20,000 ; \mathbf{d} \times 40,000 ; \mathbf{e} \times 100 ; \mathbf{f}$ $\times 100 ; \mathbf{g} \times 200$; and $\mathbf{h} \times 200$
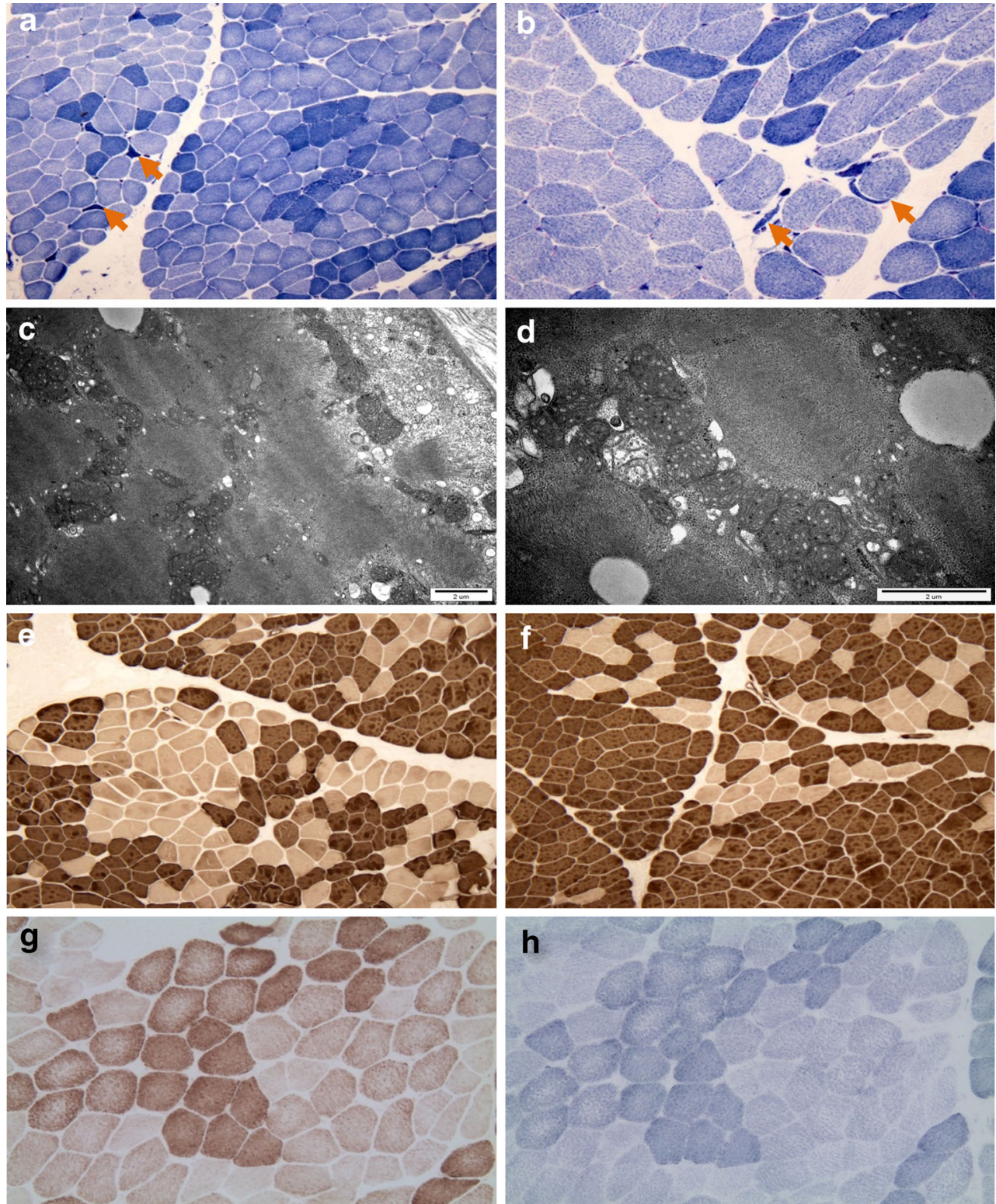

observed in patient II-1. Cerebellum and brainstem atrophy with prominent cerebellopontine cisternal spaces, a widened fourth ventricle, and an enlarged cerebellar cortical sulci were noted in both patients (Fig. 5d). However, the cerebral hemisphere and basal ganglia were normal in both patients, without evidence of atrophy or parenchymal changes.

Relatively intact muscle without abnormal signal changes or muscle atrophy was observed in the thigh (Fig. 6a, b); however, both patients exhibited hyperintense signal abnormalities with muscle atrophy in the calf (Fig. 6c, d). T1-weighted images demonstrated muscle atrophy with signal changes in the superficial posterior compartment, including the soleus and gastrocnemius muscles (arrows). Together, these findings indicate that the distal muscles are impaired more severely than the proximal muscles.

\section{Discussion}

Mutations in C10orf2 are associated with autosomal recessive IOSCA, hepatocerebral syndrome, and adPEO. Mutations cluster exclusively within the primase, linker, and helicase domains, with the majority of recessive phenotypes occurring as a result of mutations in the helicase domain [17]. In accordance with this mode of action, the two patients described here harbor novel compound heterozygous mutations in the helicase domain (p.T487I and c.1485$1 \mathrm{G}>\mathrm{A}$ ) resulting in phenotypes characteristic of IOSCA. 
Fig. 4 Histopathological characterization of distal sural nerve biopsy (patient II-2). a Gray scale image of toluidine bluestained semi-thin transverse sections revealing an absence of large MFs, along with abundant medium and small-sized MFs $\left(5,101 / \mathrm{mm}^{2}\right)$. b Histogram showing a unimodal distribution pattern of MFs with diameter $<6 \mu \mathrm{m}$, representing $97.0 \%$ of total MFs. c-d Electron micrographs revealed small MFs with excessive folding of myelin and rare axonal clusters. Original magnifications: $\mathbf{a} \times 400$; $\mathbf{c} \times 5,000$; and $\mathbf{d} \times 12,000$

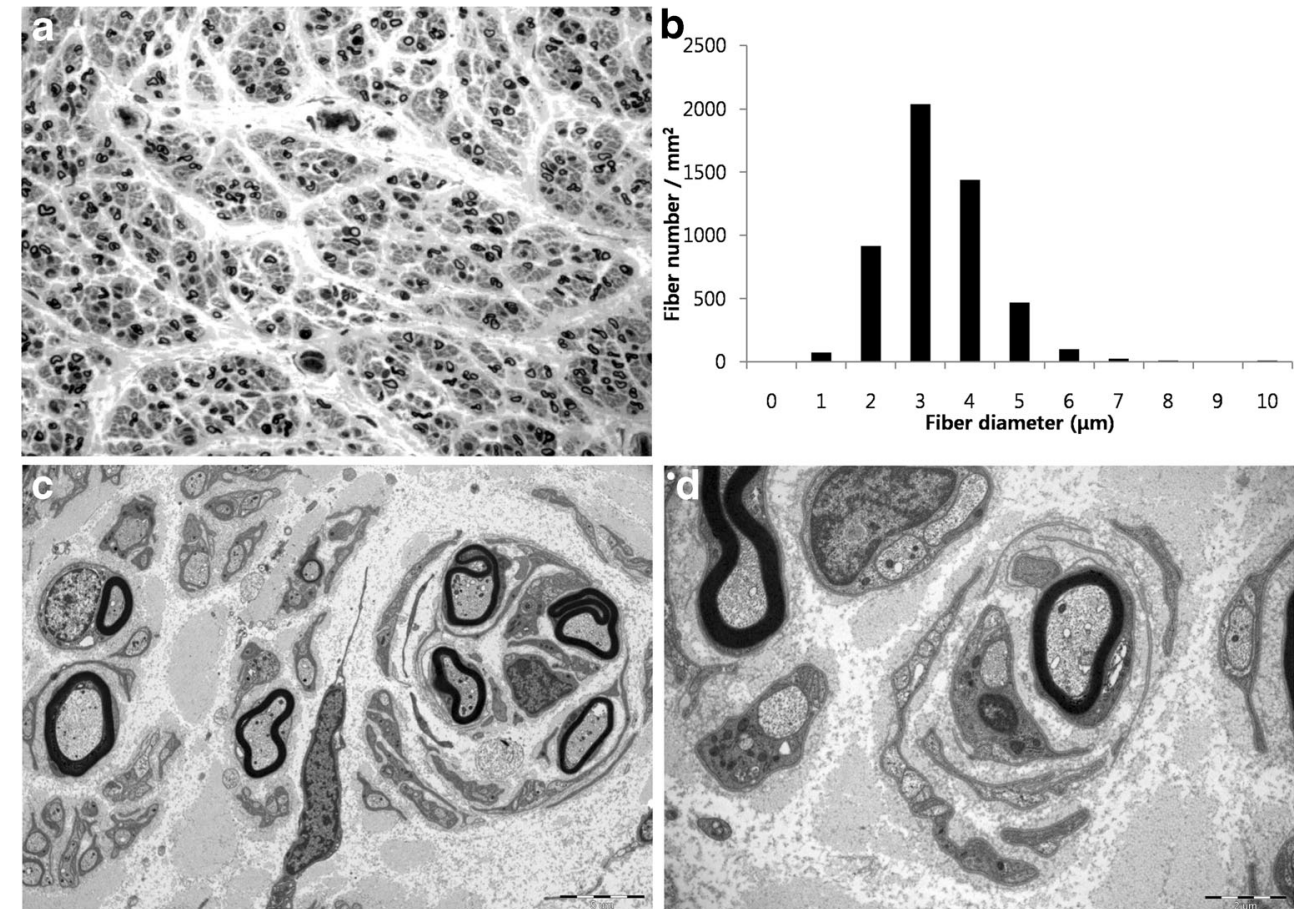

Mutations adjacent to residue T487 have been associated with both dominant and recessive phenotypes: T405I, L456V, R463W, and Y508 mutations cause IOSCA [6, 7, 18], while W474C, A475P, F478I, E479K, and F485L cause PEO [19]. The C1485-1G $>$ A mutation may result in a shorter protein, thereby resembling Twinky, a 582-amino-acid protein terminating with four unique residues. Although this study contained a small number of patients, the identified mutations were clearly validated by Sanger sequencing as well as cosegregation in the kindred (Fig. 1). In addition, neither apparent causative mutation was identified in $648 \mathrm{Ko}$ rean control samples (Table 2).

The Twinkle helicase protein, encoded by C10orf2, localizes to mitochondrial nucleoids and functions in collaboration with DNA polymerase gamma. Mutations within this protein negatively affect the integrity of mtDNA. Deletions of mtDNA are typically associated with adPEO and have not been reported in IOSCA patients [20, 21]. The identification of mtDNA deletions in this study suggests that compound mutations in the helicase domain affect C10orf2 activity in a recessive manner.

Although a few IOSCA patients have been described in Finland, only three cases have been reported elsewhere, with no cases reported in Asian populations [5-7, 10, 22, 23]. Axonal sensory neuropathy is thought to be the most important pathological feature of IOSCA; however, this report represents the first description of an IOSCA patient harboring motor neuropathy. Before referral to our clinic, the two patients described in this report were first diagnosed with hereditary motor and sensory neuropathies (HMSN) due to sensory and motor
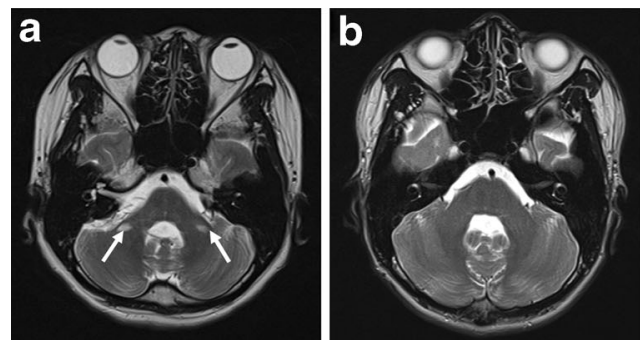

Fig. 5 Brain MRI in patient II-1 (a) and patient II-2 (b-d). a Highintensity lesions of the bilateral middle cerebellar peduncle (arrows) in T2-weighted imaging (patient II-1; 20 years of age). b At 17 years of age, no high-intensity lesions were found in the middle cerebellar peduncle of patient II-2. c However, a follow-up brain MRI 3 years later revealed
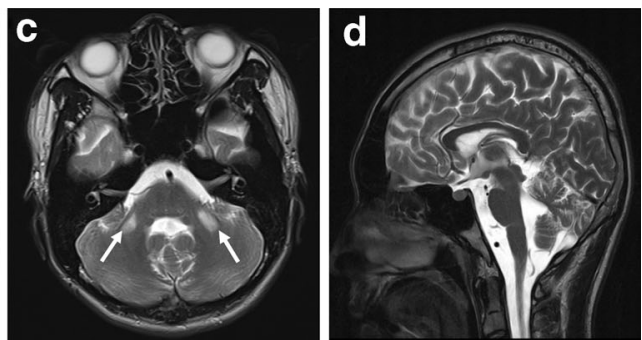

bilateral middle cerebellar peduncle lesions (arrows). d The bilateral cerebellum and brainstem were atrophied, with prominent cerebellopontine cisternal spaces, a widened fourth ventricle, and enlarged cerebellar cortical sulci were observed in patient II- 2 at 20 years of age 


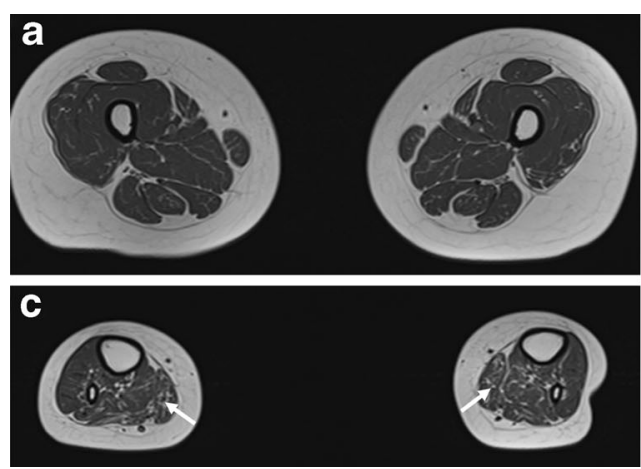

Fig. 6 Lower limb MRIs in patient II-1. MRIs of the thigh $(\mathbf{a}, \mathbf{b})$ and lower leg $(\mathbf{c}, \mathbf{d})$ at $20(\mathbf{a}, \mathbf{c})$ and $23(\mathbf{b}, \mathbf{d})$ years of age. Follow-up studies revealed progression of atrophic changes. a, b At the thigh level, T1weighted images demonstrated relatively intact muscle without signal

neuropathy and moderate to severe foot deformities including high arches. Based upon these initial observations, we believe that the phenotypic spectrum of IOSCA may be wider than reported previously. Screening patients for C10orf2 mutations may be beneficial for patients with sensorimotor polyneuropathy, particularly HMSN patients who present with symptoms of spinocerebellar ataxia.

Histopathological analysis of the muscle biopsy revealed myopathies harboring small, angulated, or elongated degenerating myofibers containing aggregated, enlarged mitochondria with concentric cristae. High ratios of lactate/ pyruvate were also observed. Although there was no evidence of ragged red fibers by Gomori's trichrome staining, or increased SDH or NADH, the presence of scattered degenerating myofibers with abnormal mitochondria suggests an association with mtDNA deletions.

Brain MRI findings from both IOSCA patients revealed high-intensity lesions of the middle cerebellar peduncles. In patient II-2, no lesions were observed at 17 years of age; however, clear bilateral cerebellar lesions were evident by age 20 years, consistent with observations of more severe ataxia. These results suggest a correlation between the progression of the clinical ataxia and the increasing severity of degenerative IOSCA. Middle peduncular lesions may be useful as a prognostic biomarker of IOSCA. MRI analysis of the lower extremities showed muscle atrophy with hyperintense signal changes in the lower leg muscles of both patients. However, signal intensities were normal in the thigh muscles, consistent with the hypothesis of length-dependent axonal degeneration.

In conclusion, we identified novel compound heterozygous mutations c. 1460C $>$ T (p.T487I) and c.1485-1G $>$ A in C10orf2 as the underlying cause of IOSCA combined with sensorimotor polyneuropathy and myopathy. This study suggests greater variability in the clinical spectrum of IOSCA than reported previously and that screening of C10orf2 may be helpful in diagnosing IOSCA, especially in patients

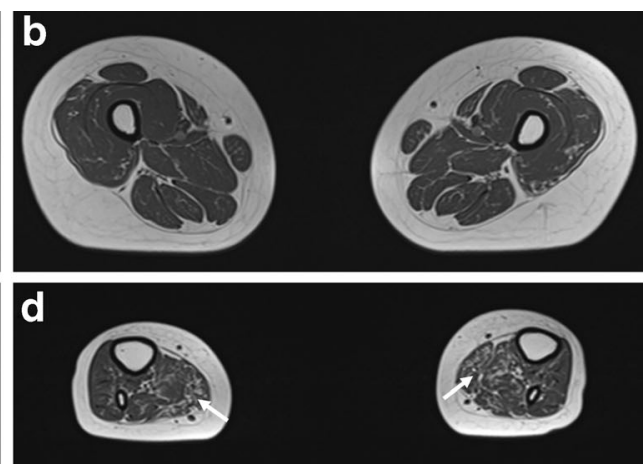

changes or atrophies. c, d In the lower leg, MRIs revealed moderate involvement of superficial posterior compartment muscles, including the soleus and gastrocnemius muscles (arrows); however, anterior and lateral compartment muscles were spared

presenting with symptoms of axonal sensorimotor polyneuropathy and myopathy.

Acknowledgments This work was supported by the Korea National Institute of Health intramural research program 4800-4845-302-210-13 (2012-N61001-00) and 4800-4861-307-210-13 (2012-NG61004-00), the Basic Science Research Program through the National Research Foundation (NRF) funded by the Ministry of Education (20110021533), and the Korean Health Technology R\&D Project, Ministry of Health and Welfare, Republic of Korea (A120182).

Conflict of interest The authors report no conflict of interest.

Open Access This article is distributed under the terms of the Creative Commons Attribution License which permits any use, distribution, and reproduction in any medium, provided the original author(s) and the source are credited.

\section{References}

1. Zeviani M, Di Donato S (2004) Mitochondrial disorders. Brain 127: 2153-2172

2. Greaves LC, Reeve AK, Taylor RW et al (2012) Mitochondrial DNA and disease. J Pathol 226:274-286

3. Park CB, Larsson NG (2011) Mitochondrial DNA mutations in disease and aging. J Cell Biol 193:809-818

4. Spelbrink JN, Li FY, Tiranti V et al (2001) Human mitochondrial DNA deletions associated with mutations in the gene encoding Twinkle, a phage T7 gene 4-like protein localized in mitochondria. Nat Genet 28:223-231

5. Koskinen T, Sainio K, Rapola J et al (1994) Sensory neuropathy in infantile onset spinocerebellar ataxia (IOSCA). Muscle Nerve 17:509-515

6. Dündar H, Ozgül RK, Yalnızoğlu D et al (2012) Identification of a novel Twinkle mutation in a family with infantile onset spinocerebellar ataxia by whole exome sequencing. Pediatr Neurol 46:172-177

7. Hartley JN, Booth FA, Del Bigio MR et al (2012) Novel autosomal recessive c10orf 2 mutations causing infantile-onset spinocerebellar ataxia. Case Rep Pediatr 2012:303096 
8. Nikali K, Suomalainen A, Saharinen J et al (2005) Infantile onset spinocerebellar ataxia is caused by recessive mutations in mitochondrial proteins Twinkle and Twinky. Hum Mol Genet 14:2981-2990

9. Van Hove JL, Cunningham V, Rice C et al (2009) Finding Twinkle in the eyes of a 71-year-old lady: a case report and review of the genotypic and phenotypic spectrum of TWINKLE-related dominant disease. Am J Med Genet A 149:861-867

10. Hakonen AH, Isohanni P, Paetau A et al (2007) Recessive Twinkle mutations in early onset encephalopathy with mtDNA depletion. Brain 130:3032-3040

11. Birouk N, LeGuern E, Maisonobe T et al (1998) X-linked CharcotMarie-Tooth disease with connexin 32 mutations: clinical and electrophysiologic study. Neurology 50:1074-1082

12. Shy ME, Blake J, Krajewski K et al (2005) Reliability and validity of the CMT neuropathy score as a measure of disability. Neurology 64 : 1209-1214

13. Choi BO, Koo SK, Park MH et al (2012) Exome sequencing is an efficient tool for genetic screening of Charcot-Marie-Tooth disease. Hum Mutat 33:1610-1615

14. Choi BO, Park MH, Chung KW et al (2013) Clinical and histopathological study of Charcot-Marie-Tooth neuropathy with a novel S90W mutation in BSCL2. Neurogenetics 14:35-42

15. Woo HM, Park HJ, Baek JI et al (2013) Whole-exome sequencing identifies MYO15A mutations as a cause of autosomal recessive nonsyndromic hearing loss in Korean families. BMC Med Genet 14:72
16. Hong D, Park SS, Ju YS et al (2011) TIARA: a database for accurate analysis of multiple personal genomes based on cross-technology. Nucleic Acids Res 39:D883-D888

17. Korhonen JA, Pande V, Holmlund T et al (2008) Structure-function defects of the TWINKLE linker region in progressive external ophthalmoplegia. J Mol Biol 377:691-705

18. Sarzi E, Goffart S, Serre V et al (2007) Twinkle helicase (PEO1) gene mutation causes mitochondrial DNA depletion. Ann Neurol 62:579-587

19. Sanchez-Martinez A, Calleja M, Peralta S et al (2012) Modeling pathogenic mutations of human Twinkle in Drosophila suggests an apoptosis role in response to mitochondrial defects. PLoS One 7:e43954

20. Suomalainen A, Majander A, Wallin M et al (1997) Autosomal dominant progressive external ophthalmoplegia with multiple deletions of mtDNA: clinical, biochemical, and molecular genetic features of the 10q-linked disease. Neurology 48:1244-1253

21. Hudson G, Deschauer M, Busse K et al (2005) Sensory ataxic neuropathy due to a novel C10Orf2 mutation with probable germline mosaicism. Neurology 64:371-373

22. Lonnqvist T, Paetau A, Valanne L et al (2009) Recessive twinkle mutations cause severe epileptic encephalopathy. Brain 132:1553-1562

23. Koskinen T, Santavuori P, Sainio K et al (1994) Infantile onset spinocerebellar ataxia with sensory neuropathy: a new inherited disease. J Neurol Sci 121(1):50-56 